Supporting Information

\title{
Diastereoselective Proton Transfer: A Route to Enantiomerically Pure Halfsandwich Rhenium Complexes
}

\author{
Frank Bock, Frank Fischer, and Wolfdieter A. Schenk* \\ Institut für Anorganische Chemie, Bayerische Julius-Maximilians-Universität Würzburg, \\ Am Hubland, D-97074 Würzburg, Germany.
}




\section{General considerations}

All manipulations were conducted under an atmosphere of dry nitrogen or argon using standard Schlenk techniques. Solvents were purified by distillation from sodium-potassium alloy (hexane, benzene, toluene, diethylether, THF) or $\mathrm{P}_{4} \mathrm{O}_{10}\left(\mathrm{CH}_{2} \mathrm{Cl}_{2}\right)$ and stored under an atmosphere of dinitrogen. Deuterated solvents were degassed and stored over molecular sieves. IR spectra were recorded with a Bruker IFS 25 instrument set at $4 \mathrm{~cm}^{-1}$ resolution. NMR spectra were recorded with a Bruker AMX 400 spectrometer, chemical shifts are referenced to TMS $\left({ }^{1} \mathrm{H},{ }^{13} \mathrm{C}\right)$ and $85 \% \mathrm{H}_{3} \mathrm{PO}_{4}\left({ }^{31} \mathrm{P}\right)$, with the deuterium signal of the solvent serving as internal lock and the residual solvent signals as additional reference. Microanalyses were performed by the analytical laboratory of the Institut für Anorganische Chemie using a Leco CHNS-932 instrument.

\section{Syntheses}

$\left[\mathbf{C p R e}(\mathbf{N O})(\mathbf{C O})\left\{\mathbf{P}(\mathrm{Me})(\mathbf{P h})\left(\mathbf{2}-\mathbf{C}_{6} \mathbf{H}_{4} \mathbf{N M e}_{2}\right)\right\}\right] \mathrm{BF}_{4}:[\mathrm{CpRe}(\mathrm{NO})(\mathrm{CO})(\mathrm{NCMe})] \mathrm{BF}_{4}{ }^{1}(1.00 \mathrm{~g}$, $2.29 \mathrm{mmol})$ and $\mathrm{P}(\mathrm{Me})(\mathrm{Ph})\left(2-\mathrm{C}_{6} \mathrm{H}_{4} \mathrm{NMe}_{2}\right)^{2}(0.83 \mathrm{~g}, 3.40 \mathrm{mmol})$ were combined in a small flask and heated under a dynamic vacuum to $80{ }^{\circ} \mathrm{C}$ for $4 \mathrm{~h}$. The resulting solid was taken up in THF $(20 \mathrm{~mL})$ and stirred until a brownish-yellow material had separated. This was filtered off, washed repeatedly with THF, and dried under vacuum. Yield 1.36 g (93\%), m.p. $96{ }^{\circ} \mathrm{C}$. ${ }^{1} \mathrm{H}$ NMR $\left(\mathrm{CD}_{2} \mathrm{Cl}_{2}, 400 \mathrm{MHz}\right): \delta=2.17,2.21\left(\mathrm{~s}, 6 \mathrm{H} ; \mathrm{N}\left(\mathrm{CH}_{3}\right)_{2}\right), 2.52,2.56(\mathrm{~d}, J=10.0 \mathrm{~Hz}, 3 \mathrm{H}$; $\left.\mathrm{PCH}_{3}\right), 5.84,5.88\left(\mathrm{~d}, J=0.5 \mathrm{~Hz}, 5 \mathrm{H} ; \mathrm{C}_{5} \mathrm{H}_{5}\right), 7.40-7.70\left(\mathrm{~m}, 9 \mathrm{H}\right.$; aryl); ${ }^{31} \mathrm{P} \mathrm{NMR}\left(\mathrm{CD}_{2} \mathrm{Cl}_{2}\right.$, $162 \mathrm{MHz}): \delta=-10.8,-10.0(\mathrm{~s}) ; \mathrm{IR}\left(\mathrm{CH}_{2} \mathrm{Cl}_{2}\right): \mathrm{v}=2019(\mathrm{CO}), 1763(\mathrm{NO}) \mathrm{cm}^{-1}$.

$\left[\mathrm{CpRe}(\mathrm{NO})\left\{\mathrm{P}(\mathrm{Me})(\mathrm{Ph})\left(2-\mathrm{C}_{6} \mathrm{H}_{4} \mathrm{NMe}_{2}\right)\right\}\left(\mathrm{CH}_{3}\right)\right] \quad$ (1): $\quad$ To a suspension of $\left[\mathrm{CpRe}(\mathrm{NO})(\mathrm{CO})\left\{\mathrm{P}(\mathrm{Me})(\mathrm{Ph})\left(2-\mathrm{C}_{6} \mathrm{H}_{4} \mathrm{NMe}_{2}\right)\right\}\right] \mathrm{BF}_{4}(1.30 \mathrm{~g}, 2.10 \mathrm{mmol})$ in THF $(15 \mathrm{~mL})$ $\mathrm{NaBH}_{4}(0.25 \mathrm{~g}, 6.60 \mathrm{mmol})$ was added with stirring. Gas evolution ceased after $1.5 \mathrm{~h}$ while the color of the reaction mixture turned to a brownish-red. The mixture was filtered over celite, the filtrate evaporated to dryness, and the residue recrystallized from benzene/hexane. Yield 0.84 g (74\%), m.p. $154{ }^{\circ} \mathrm{C} .{ }^{1} \mathrm{H}$ NMR $\left(\mathrm{C}_{6} \mathrm{D}_{6}, 400 \mathrm{MHz}\right): \delta=1.22,1.27(\mathrm{~d}, J=6.3 \mathrm{~Hz}$, $\left.3 \mathrm{H} ; \mathrm{ReCH}_{3}\right), 1.91,2.08$ (d, J = 8.5 Hz, 3H; $\left.\mathrm{PCH}_{3}\right), 2.02,2.11\left(\mathrm{~s}, 6 \mathrm{H} ; \mathrm{N}\left(\mathrm{CH}_{3}\right)_{2}\right), 4.65,4.67$ (s, $\left.5 \mathrm{H} ; \mathrm{C}_{5} \mathrm{H}_{5}\right), 6.92-7.76\left(\mathrm{~m}, 9 \mathrm{H}\right.$; aryl); ${ }^{13} \mathrm{C} \mathrm{NMR}\left(\mathrm{C}_{6} \mathrm{D}_{6}, 100 \mathrm{MHz}\right): \delta=-38.6(\mathrm{~d}, J=7 \mathrm{~Hz}$, $\left.\mathrm{ReCH}_{3}\right),-38.2\left(\mathrm{~d}, J=7 \mathrm{~Hz}, \mathrm{ReCH}_{3}\right), 17.2\left(\mathrm{~d}, J=36 \mathrm{~Hz}, \mathrm{PCH}_{3}\right), 18.2\left(\mathrm{~d}, J=33 \mathrm{~Hz}, \mathrm{PCH}_{3}\right)$, $45.6\left(\mathrm{~s}, \mathrm{NCH}_{3}\right), 46.1\left(\mathrm{~s}, \mathrm{NCH}_{3}\right), 88.3\left(\mathrm{~d}, J=2 \mathrm{~Hz}, \mathrm{C}_{5} \mathrm{H}_{5}\right), 88.5\left(\mathrm{~d}, J=2 \mathrm{~Hz}, \mathrm{C}_{5} \mathrm{H}_{5}\right) ;{ }^{31} \mathrm{P} \mathrm{NMR}$ $\left(\mathrm{C}_{6} \mathrm{D}_{6}, 162 \mathrm{MHz}\right): \delta=0.7,2.4(\mathrm{~s})$; IR $\left(\mathrm{CH}_{2} \mathrm{Cl}_{2}\right): v=1636(\mathrm{NO}) \mathrm{cm}^{-1}$. Elemental analyses: calcd for $\mathrm{C}_{21} \mathrm{H}_{26} \mathrm{~N}_{2} \mathrm{OPRe}$ : C 46.74, H 4.86, N 5.19; found: C 46.96, H 4.72, N 5.08. 
$\left[\mathrm{CpRe}(\mathrm{NO})\left\{\mathbf{P}(\mathrm{Me})(\mathbf{P h})\left(\mathbf{2}-\mathrm{C}_{6} \mathrm{H}_{4} \mathbf{N M e}_{2}\right)\right\}\right] \mathrm{BF}_{4}$, diastereomeric mixture: To a solution of $\mathbf{1}$ $(0.50 \mathrm{~g}, 0.93 \mathrm{mmol})$ in $\mathrm{CH}_{2} \mathrm{Cl}_{2}(15 \mathrm{~mL}), \mathrm{HBF}_{4}$ in diethylether $(93 \mu \mathrm{L}, 1.25 \mathrm{mmol})$ was added at $-78{ }^{\circ} \mathrm{C}$. The mixture was stirred at this temperature for $1 \mathrm{~h}$ and then allowed to slowly warm up to $20^{\circ} \mathrm{C}$. After gas evolution had ceased the mixture was evaporated to $5 \mathrm{~mL}$ and the product precipitated by adding diethylether $(10 \mathrm{~mL})$. The supernatant was removed by syringe, and the residue washed twice with diethylether and hexane and dried under vacuum. Yield 0.52 g (91\%), m.p. $196{ }^{\circ} \mathrm{C}$ (dec.). ${ }^{1} \mathrm{H}$ NMR $\left(\mathrm{CD}_{2} \mathrm{Cl}_{2}, 400 \mathrm{MHz}\right): \delta=2.30,(\mathrm{~d}, J=9.9$ $\left.\mathrm{Hz}, 3 \mathrm{H} ; \mathrm{PCH}_{3}\right), 2.45$, (d, $\left.J=10.8 \mathrm{~Hz}, 3 \mathrm{H} ; \mathrm{PCH}_{3}\right), 3.10$ (s, 3H; $\left.\mathrm{NCH}_{3}\right), 3.16\left(\mathrm{~s}, 3 \mathrm{H} ; \mathrm{NCH}_{3}\right)$, $4.18\left(\mathrm{~s}, 3 \mathrm{H} ; \mathrm{NCH}_{3}\right), 4.25\left(\mathrm{~s}, 3 \mathrm{H} ; \mathrm{NCH}_{3}\right), 5.46\left(\mathrm{~s}, 5 \mathrm{H} ; \mathrm{C}_{5} \mathrm{H}_{5}\right), 5.77\left(\mathrm{~s}, 5 \mathrm{H} ; \mathrm{C}_{5} \mathrm{H}_{5}\right), 7.22$ - 7.94 (m, 18H; aryl); ${ }^{31} \mathrm{P}$ NMR $\left(\mathrm{CD}_{2} \mathrm{Cl}_{2}, 162 \mathrm{MHz}\right): \delta=10.6$ (s), 11.9 (s); IR $\left(\mathrm{CH}_{2} \mathrm{Cl}_{2}\right): \mathrm{v}=1697$ (NO) $\mathrm{cm}^{-1}$. Elemental analyses: calcd for $\mathrm{C}_{20} \mathrm{H}_{23} \mathrm{BF}_{4} \mathrm{~N}_{2}$ OPRe: C 39.29, H 3.79, N 4.58; found: C $38.73, \mathrm{H} 3.75, \mathrm{~N} 4.45$.

Unlike $-\left[\mathrm{CpRe}(\mathrm{NO})\left\{\mathrm{P}(\mathrm{Me})(\mathrm{Ph})\left(2-\mathrm{C}_{6} \mathrm{H}_{4} \mathrm{NMe}_{2}\right)\right\}\right] \mathrm{MeSO}_{3}$ (unlike-2): To a solution of $\mathbf{1}$ (100 $\mathrm{mg}, 0.185 \mathrm{mmol})$ in $\mathrm{CH}_{2} \mathrm{Cl}_{2}(5 \mathrm{~mL}), \mathrm{MeSO}_{3} \mathrm{H}(6.2 \mu \mathrm{L}, 0.095 \mathrm{mmol})$ was added at $-78{ }^{\circ} \mathrm{C}$. The reaction mixture was worked up as described above giving unlike-2 as a pale yellow solid. Yield $0.53 \mathrm{mg}(93 \%)$, m.p. $197{ }^{\circ} \mathrm{C}$ (dec.). ${ }^{1} \mathrm{H}$ NMR $\left(\mathrm{CD}_{2} \mathrm{Cl}_{2}, 400 \mathrm{MHz}\right): \delta=2.38,(\mathrm{~d}, J$ $\left.=10.3 \mathrm{~Hz}, 3 \mathrm{H} ; \mathrm{PCH}_{3}\right), 2.50,\left(\mathrm{~s}, 3 \mathrm{H} ; \mathrm{O}_{3} \mathrm{SCH}_{3}\right), 3.24\left(\mathrm{~s}, 3 \mathrm{H} ; \mathrm{NCH}_{3}\right), 4.21\left(\mathrm{~s}, 3 \mathrm{H} ; \mathrm{NCH}_{3}\right), 5.87$ (s, 5H; $\left.\mathrm{C}_{5} \mathrm{H}_{5}\right), 7.43-7.90$ (m, 9H; aryl); ${ }^{31} \mathrm{P}$ NMR $\left(\mathrm{CD}_{2} \mathrm{Cl}_{2}, 162 \mathrm{MHz}\right): \delta=11.0$ (s); IR $\left(\mathrm{CH}_{2} \mathrm{Cl}_{2}\right): \mathrm{v}=1698(\mathrm{NO}) \mathrm{cm}^{-1}$.

Like- $\left[\mathrm{CpRe}(\mathrm{NO})\left\{\mathrm{P}(\mathrm{Me})(\mathrm{Ph})\left(2-\mathrm{C}_{6} \mathrm{H}_{4} \mathrm{NMe}_{2}\right)\right\}\right] \mathrm{MeSO}_{3}$ (like-2): The supernatant containing like-1 was evaporated to dryness, the residue dissolved in $\mathrm{CH}_{2} \mathrm{Cl}_{2}(5 \mathrm{~mL})$ and treated with a slight excess of $\mathrm{MeSO}_{3} \mathrm{H}$ as described above. Workup as usual gave like-2 as a pale yellow solid. Yield $0.50 \mathrm{mg}(87 \%)$, m.p. $152{ }^{\circ} \mathrm{C}$ (dec.). ${ }^{1} \mathrm{H} \mathrm{NMR}\left(\mathrm{CD}_{2} \mathrm{Cl}_{2}, 400 \mathrm{MHz}\right): \delta=2.45,(\mathrm{~d}, J$ $\left.=10.9 \mathrm{~Hz}, 3 \mathrm{H} ; \mathrm{PCH}_{3}\right), 2.59$, (s, 3H; $\left.\mathrm{O}_{3} \mathrm{SCH}_{3}\right), 3.17\left(\mathrm{~s}, 3 \mathrm{H} ; \mathrm{NCH}_{3}\right), 4.29\left(\mathrm{~s}, 3 \mathrm{H} ; \mathrm{NCH}_{3}\right), 5.54$ (s, 5H; $\left.\mathrm{C}_{5} \mathrm{H}_{5}\right), 7.44-8.06$ (m, 9H; aryl); ${ }^{31} \mathrm{P}$ NMR $\left(\mathrm{CD}_{2} \mathrm{Cl}_{2}, 162 \mathrm{MHz}\right): \delta=11.8$ (s); IR $\left(\mathrm{CH}_{2} \mathrm{Cl}_{2}\right): v=1698(\mathrm{NO}) \mathrm{cm}^{-1}$.

$(\boldsymbol{R})-\mathbf{P}(\mathrm{Me})(\mathbf{P h})\left(2-\mathrm{C}_{6} \mathbf{H}_{4} \mathbf{N M e}_{2}\right)$ : To a freshly prepared solution of $(S)-\mathrm{P}(\mathrm{Cl})(\mathrm{Me})(\mathrm{Ph})\left(\mathrm{BH}_{3}\right)^{3}$ $(6.64 \mathrm{mmol})$ in toluene $(300 \mathrm{~mL})$ was added at $-78{ }^{\circ} \mathrm{C}$ a suspension of $\mathrm{Li}\left(2-\mathrm{C}_{6} \mathrm{H}_{4} \mathrm{NMe}_{2}\right)^{4}$ (ca. $16 \mathrm{mmol})$. The mixture was slowly warmed up to $20^{\circ} \mathrm{C}$ and quenched with water $(80 \mathrm{~mL})$. The organic layer was separated, the aqueous phase extracted twice with dichloromethane (50 
$\mathrm{mL}$ ), and the combined organic phases evaporated to dryness. The residue was dissolved in toluene $(20 \mathrm{~mL})$, filtered over silica, and evaporated again, leaving $(R)-\mathrm{P}(\mathrm{Me})(\mathrm{Ph})(2-$ $\left.\mathrm{C}_{6} \mathrm{H}_{4} \mathrm{NMe}_{2}\right)\left(\mathrm{BH}_{3}\right)$ as a colorless viscous oil. Yield $1.36 \mathrm{~g}(80 \%) .{ }^{1} \mathrm{H} \mathrm{NMR}\left(\mathrm{CD}_{2} \mathrm{Cl}_{2}, 300\right.$ MHz): $\delta=1.94,\left(\mathrm{~d}, J=10.1 \mathrm{~Hz}, 3 \mathrm{H} ; \mathrm{PCH}_{3}\right), 2.20$, (s, 6H; $\left.\mathrm{N}\left(\mathrm{CH}_{3}\right)_{2}\right), 6.65-7.83$ (m, 9H; aryl); ${ }^{31} \mathrm{P}$ NMR $\left(\mathrm{CD}_{2} \mathrm{Cl}_{2}, 121.5 \mathrm{MHz}\right): \delta=8.2$ (q, $J=62 \mathrm{~Hz}$,). This material was dissolved in diethylamine $(20 \mathrm{~mL})$ and kept at $50{ }^{\circ} \mathrm{C}$ for $2.5 \mathrm{~h}$. Excess amine was removed under vacuum and the residue dissolved in pentane $(20 \mathrm{~mL})$ and filtered over basic alumina. Evaporation left $(R)-\mathrm{P}(\mathrm{Me})(\mathrm{Ph})\left(2-\mathrm{C}_{6} \mathrm{H}_{4} \mathrm{NMe}_{2}\right)$ in $92 \%$ ee $\left({ }^{1} \mathrm{H}\right.$ NMR in the presence of $(R)-(-)$-TFAE) as a colorless viscous oil. Yield $1.26 \mathrm{~g}(98 \%) .{ }^{1} \mathrm{H}$ NMR $\left(\mathrm{CD}_{2} \mathrm{Cl}_{2}, 300 \mathrm{MHz}\right): \delta=1.54,(\mathrm{~d}, J=4.9$ $\left.\mathrm{Hz}, 3 \mathrm{H} ; \mathrm{PCH}_{3}\right), 2.62$, (s, 6H; N( $\left.\left(\mathrm{CH}_{3}\right)_{2}\right), 6.64-7.43$ (m, 9H; aryl); ${ }^{31} \mathrm{P} \mathrm{NMR}\left(\mathrm{CD}_{2} \mathrm{Cl}_{2}, 121.5\right.$ $\mathrm{MHz}): \delta=-35.1(\mathrm{~s})$.

$\left(R_{\mathrm{Re}}, S_{\mathrm{P}}\right)-\left[\mathrm{CpRe}(\mathrm{NO})\left\{\mathrm{P}(\mathrm{Me})(\mathrm{Ph})\left(2-\mathrm{C}_{6} \mathrm{H}_{4} \mathrm{NMe}_{2}\right)\right\}\right] \mathrm{MeSO}_{3} \quad\left(\left(R_{\mathrm{Re}}, S_{\mathrm{P}}\right)-2\right) \quad$ and $\quad\left(S_{\mathrm{Re}}, S_{\mathrm{P}}\right)-$ $\left[\mathrm{CpRe}(\mathrm{NO})\left\{\mathrm{P}(\mathrm{Me})(\mathrm{Ph})\left(2-\mathrm{C}_{6} \mathrm{H}_{4} \mathrm{NMe}_{2}\right)\right\}\right] \mathrm{MeSO}_{3} \quad\left(\left(S_{\mathrm{Re}}, S_{\mathrm{P}}\right)-2\right)$ : Repetition of the above sequence with the use of $(R)-\mathrm{P}(\mathrm{Me})(\mathrm{Ph})\left(2-\mathrm{C}_{6} \mathrm{H}_{4} \mathrm{NMe}_{2}\right)$ gave the two diastereoisomers in enantiomerically pure form as described in the main text.

\section{References}

(1) Agbossou, F.; O'Connor, E. J.; Garner, C. M., Mendez, N. Q.; Fernandez, J. M.; Patton, A. T.; Ramsden, J. A.; Gladysz, J. A. Inorg. Synth. 1992, 29, 211.

(2) Horner, L.; Simons, G. Phosphorus and Sulfur 1984, 19, 77.

(3) Bauduin, C.; Moulin, D.; Kaloun, E. B.; Darcel, C.; Jugé, S.; J. Org. Chem. 2003, 68, 4293.

(4) Slocum, D. W.; Book, G.; Jennings, C. A.; Tetrahedron Lett. 1970, 39, 3443. 